\title{
The Extended Supersymmetrization of the Nonlinear Schrödinger Equation
}

\author{
Ziemowit Popowicz \\ Institute of Theoretical Physics, University of Wroclaw \\ pl. M. Borna 9, 50-205 Wroclaw Poland \\ e-mail ZIEMEK @ PLWRUW11.BITNET
}

\begin{abstract}
We present the new extended supersymmetrization of the Nonlinear Schrödinger Equation by introducing two superbosons fields with the different gradation. Our model is different from this considered by Toppan and in the reduced case (nonextended supersymmetry) is also different from this considered by Kulish and Roelofs and Kersten. We prove that our model is integrable by presenting its Lax formulation.
\end{abstract}

\section{Introduction}

The nonlinear solitonic equations have been widely studied since the fundamental work of Gardner, Green, Kruskal and Miura [1-4] on the Korteweg-de Vries equation. It appeared that such equations describe a large number of physical phenomenons and hence all possible generalizations leading to soliton's behaviour were found to be are of great importance. Interestingly the supersymmetrization gives us an alternative manner of generalization [5].

The main idea of the supersymmetrization, which comes from quantum theory is to unify bosons and fermions. While connecting supersymmetry with nonlinear equations, one should distinguish between two classes of supersymmetry - the socalled extended and nonextended supersymmetry. In the nonextended case, one generalizes the given function which describes the soliton behaviour by the addition of one (or several) fermionic fields. As a result it was possible to generalize [68] many soliton equations and obtain symmetries, conservation laws, prolongation structure, Lax pair and its bihamiltonian structure. However in this generalization we encounter the problem of the interpretation of new fermionic fields on the classical level. On the other hand this effort is not an academic problem only. The knowledge of the nonextended supersymmetrization allows us, in many cases to produce the extended supersymmetrization. 
We include in this case in addition to fermions, also new bosons fields. After the supersymmetrization it appears that the so-called bosonic part of the model (in which all fermions vanish) give us the new generalization of the model. In such manner, several soliton's equation have been supersymmetrized, $K d V$ equation [811] and Boussinesq equation [12-13] as examples.

In contrast, the supersymmetrization of the Nonlinear Schrödinger Equation [7, 14-17] has not drawn as much attention. In fact, it was only recently that the nonextended supersymmetrization of the Nonlinear Schrödinger Equation was proposed $[7,14-16]$. In contrast to the supersymmet KdV equation it appeared that there exist only one supersymmetric extension of this equation which is integrable.

Recently Toppan [17] has shown that the second hamiltonian structure of the Nonlinear Schrödinger Equation is connected with the reduced $\operatorname{sl}(2)$ Kac-Moody algebra (in the Dirac sense). This observation allowed him to obtain the nonextended supersymmetrization of the Nonlinear Schrödinger Equation. However for the extended case $(\mathrm{N}=2)$ there are no supersymmetric extensions of the Kac-Moody algebra [8] and therefore Toppan has proposed to use two copies of chiral and antichiral $(\mathrm{N}=1)$ supercurrents in order to construct the analog of the extended supersymmetric version of the Nonlinear Schrödinger Equation.

In this paper we present new extended supersymmetrization of the Nonlinear Schrödinger Equation. Our idea is based on the introduction of tw supermultiplets with the different gradation. Namely, we introduce two supercurrents of the gradation one and zero respectively. Only in the special bosonic limit (described below) we recover the usual Nonlinear Schrödinger Equation. Our model is different then this considered by Toppan Moreover after reducing our model to the nonextended case we obtain the model which is different from the one considered by Kulish [7], Roelofs and Kersten [14] and by Brunelli and Das [16]. Our model in this nonextended case corresponds to the missing possibilities in the Roelofs and Kersten discussion - to the mixing gradation of the fermion fields.

All results presented in this paper were obtained due to the extended application of the symbolic computer language REDUCE - namely the Lax pair for our model. The existence of such pair proves that our model is integrable and yields the presentation of conservation laws together with its first supersymmetric hamiltonian structure.

\section{Supersymmetric Nonlinear Schrödinger Equation}

The basic object in the supersymmetric analysis is the superfield and the supersymmetric derivative. The superfields are the superfermions or the superbosons depending, in addition to $x$ and $t$, upon two anticommuting variables, $\Theta_{1}$ and $\Theta_{2}$ $\left(\Theta_{2} \Theta_{1}=-\Theta_{1} \Theta_{2}, \Theta_{1}^{2}=\Theta_{2}^{2}=0\right)$. Its Taylor expansion with respect to $\Theta^{\prime}$ 's is

$$
\Phi\left(x, \Theta_{1}, \Theta_{2}\right)=\omega(x)+\Theta_{1} \zeta_{1}(x)+\Theta_{2} \zeta_{2}(x)+\Theta_{2} \Theta_{1} u(x),
$$

where the fields $w, u$ are to be interpreted as the bosons (fermions) fields while $\zeta_{1}, \zeta_{2}$ as the fermions (bosons) for the superbosons (superfermions) field $\Phi$. The 
superderivatives are defined as

$$
\mathcal{D}_{1}=\partial_{\Theta_{1}}+\Theta_{1} \partial_{x}, \quad \mathcal{D}_{2}=\partial_{\Theta_{2}}+\Theta_{2} \partial_{x}
$$

with the properties

$$
\begin{gathered}
\mathcal{D}_{2} \mathcal{D}_{1}+\mathcal{D}_{1} \mathcal{D}_{2}=0, \\
\mathcal{D}_{1}^{2}=\mathcal{D}_{1}^{2}=\partial_{x} .
\end{gathered}
$$

Below we shall use the following notation $\left(\mathcal{D}_{i} \phi\right)$ denotes the outcome of the action of the superderivative on the superfield, while $\mathcal{D}_{i} \Phi$ denotes the action itself of the superderivative on the superfield. We use in our construction two superboson fields

$$
\begin{aligned}
& F=j_{+}+\Theta_{1} \zeta_{1}+\Theta_{2} \zeta_{2}+\Theta_{2} \Theta_{1} f, \\
& G=g+\Theta_{1} \eta_{1}+\Theta_{2} \eta_{2}+\Theta_{2} \Theta_{1} j_{-} .
\end{aligned}
$$

Our construction heavily relies on the gradation of (6) and (7). We choose zero as the gradation for $G$ while the one for $F$ with the following prescription

$$
\begin{array}{lll}
\operatorname{deg}(x)=-1, & \operatorname{deg}(t)=-2, & \operatorname{deg}(\Theta)=-1 / 2, \\
\operatorname{deg}\left(j_{+}\right)=1, & \operatorname{deg}(\zeta)=3 / 2, & \operatorname{deg}(f)=2, \\
\operatorname{deg}\left(j_{-}\right)=1, & \operatorname{deg}(\eta)=1 / 2, & \operatorname{deg}(g)=0 .
\end{array}
$$

The extended supersymmetric Nonlinear Schrödinger Equation which we would like to study has the form

$$
\begin{gathered}
\dot{F}=F_{x x}-G^{2} F^{3}+2 F\left(\mathcal{D}_{1} \mathcal{D}_{2}(G F)\right), \\
\dot{G}=-G_{x x}+G^{3} F^{2}-2 G\left(\mathcal{D}_{1} \mathcal{D}_{2}(G F)\right),
\end{gathered}
$$

where the dot denotes the time derivative while $x$ the space derivative.

Notice that the superboson $G$ has different gradation than $F$, hence our equation (10-11) could not be written as a single equation. In other words the superboson $G$ is not complex conjugate to superboson $F$. On the other hand these equations can be written in the component form using (5-6), but the formulas are complicated. We can investigate the properties of our generalization and we present two particular reductions, from which we obtain the usual Schrödinger equation:

a. The bosonic limit in which all fermions vanish

$$
\begin{aligned}
\dot{j_{+}}= & j_{+x x}-j_{+}^{3} g^{2}-2 j_{+} g f+2 j_{+}^{2} j_{-}, \\
\dot{f}= & f_{x x}+2 g f^{2}-2 j_{+x x} j_{-} g-4 j_{+x} j_{+} g_{x} \\
& -2 j_{+}^{2} g_{x x}-3 j_{+}^{2} g^{2} f-2 j_{-} j_{+}^{3} g+2 j_{-} j_{+} f,
\end{aligned}
$$




$$
\begin{aligned}
\dot{g}= & -g_{x x}-2 g^{2} f+j_{+}^{2} g^{3}-2 j_{-} j_{+} g, \\
\dot{j_{-}}= & -j_{-x x}+2 j_{-x x} g^{2}+4 j_{-x} g_{x} g+2 j_{-} g_{x x} g+ \\
& 2 j_{-} g^{3} f-2 j_{-}^{2} j_{+}-2 j_{-} g f+3 j_{-} j_{+}^{2} g^{2} .
\end{aligned}
$$

Moreover these equations could be reduced further by demanding that $f=0$ and $g=0$ or $j_{+}=0$ and $j_{-}=0$. In the first case we obtain the usual Nonlinear Schrödinger Equation

$$
\begin{aligned}
& \dot{j_{+}}=j_{+x x}+2 j_{+}^{2} j_{-}, \\
& \dot{j_{-}}=-j_{-x x}-2 j_{-}^{2} j_{+},
\end{aligned}
$$

assuming that $j_{-}^{*}=j_{+}$where $*$ denotes the complex conjugation. In the second case we obtain the following equations

$$
\begin{gathered}
\dot{f}=f_{x x}+2 g f^{2}, \\
\dot{g}=-g_{x x}-2 g^{2} f,
\end{gathered}
$$

which are similar to the equations (16) and (17). However these could not be interpreted as the Nonlinear Schrödinger Equation because $f$ is not complex conjugate to $g$ due to the different gradation.

b. Nonextended case is obtained assuming that

$$
\begin{gathered}
F=F_{1}=j_{+}+\Theta_{1} \zeta, \\
G=\Theta_{2} G_{1}=\Theta_{2}\left(\eta+\Theta_{1} j_{-}\right),
\end{gathered}
$$

which reduces our equations (10-11) to

$$
\begin{gathered}
\dot{F}_{1}=F_{1 x x}+2 F_{1}\left(\mathcal{D}_{1}\left(G_{1} F_{1}\right)\right), \\
\dot{G}_{1}=-G_{1 x x}-2 G_{1}\left(\mathcal{D}_{1}\left(G_{1} F_{1}\right)\right) .
\end{gathered}
$$

In the component these equations take the form

$$
\begin{aligned}
& \dot{j_{+}}=j_{+x x}+2 j_{+}^{2} j_{-}+2 j_{+} \zeta \eta \\
& \dot{\zeta}=\zeta_{x x}+2 j_{+}\left(j_{+} \eta\right)_{x}+2 \zeta j_{+} j_{-}, \\
& \dot{\eta}=-\eta_{x x}-2 \eta j_{+} j_{-}, \\
& \dot{j_{-}}=-j_{x x}-2 j_{-}^{2} j_{+}-2 \zeta \eta j_{-}-2 \eta \eta_{x} j_{x},
\end{aligned}
$$

from which it immediately follows that our equations are different from these considered by Kulish [7], Roelofs and Kersten [14]. 


\section{The Lax and Hamiltonian Formulations and Conservation Laws}

The Nonlinear Schrödinger Equation has been solved via the inverse scattering transformation by Zakharov and Shabat [18]. Later, it appeared that this equation could be solved in the scheme of the AKNS method [4]. Quite recently it appeared that this equation could be formulated in terms of the scalar as well as matrix Lax pair [16-17]. The Nonlinear Schrödinger Equation is the bihamiltonian equation e.q. it can be written down as

$$
\frac{d}{d t}\left(\begin{array}{l}
j_{+} \\
j_{-}
\end{array}\right)=P_{2} \cdot \operatorname{grd} H_{2}=P_{1} \cdot \operatorname{grd} H_{3},
$$

where grd denotes the gradient of the functional

$$
\begin{aligned}
& H_{3}=\int d x\left(J_{+x} J_{-x}+J_{+}^{2} J_{-}^{2}\right), \\
& H_{2}=\int d x J_{+x} J_{-},
\end{aligned}
$$

while the $P$ is the Poisson tensor

$$
\begin{gathered}
P_{1}=\left(\begin{array}{cc}
0 & 1 \\
-1 & 0
\end{array}\right), \\
P_{2}=\left(\begin{array}{cc}
-2 J_{+} \partial^{-1} J_{+}, & \partial_{x}+2 J_{+} \partial^{-1} J_{-} \\
\partial_{x}+2 J_{-} \partial^{-1} J_{+}, & -2 J_{-} \partial^{-1} J_{-}
\end{array}\right) .
\end{gathered}
$$

The $P_{2}$ operator could be obtained from the Kac-Moody $s l(2)$ algebra, if we use the Dirac reduction procedure. Indeed let $P$

$$
P=\left(\begin{array}{ccc}
0 & \partial_{x}-2 J_{0} & -J_{+} \\
\partial_{x}+2 J_{0} & 0 & J_{-} \\
J_{+} & -J_{-} & -\frac{1}{2} \partial_{x}
\end{array}\right),
$$

be a Poisson tensor which corresponds to the Kac-Moody algebra. Now let us briefly explain the standard Dirac reduction [19] formula. Let $U, V$ be two linear spaces with coordinates $u$ and $v$. Let

$$
P(u, v)=\left[\begin{array}{cc}
P_{u u}, & P_{u v} \\
P_{v u}, & P_{v v}
\end{array}\right],
$$

be a Poisson tensor on $U \oplus V$. Assume that $P_{v v}$ is invertible, then

$$
P=P_{u u}-P_{u v} P_{v v}^{-1} P_{v u}
$$


is a Poisson tensor on $U$. Now it is easy to confirm yourself that the application of the formula (35) to the $s l(2)$ Kac-Moody algebra yields the formula (32).

In the next we will consider the scalar Lax pair of the Nonlinear Schrödinger Equation. To state the problem, let us first briefly describe the KP hierarchy following [2] and the convention introduced there. The pseudodifferential Lax operator is defined by

$$
L=\partial_{x}+\sum_{i=0}^{\infty} U_{i} \partial^{-i},
$$

where the $u_{i}$ are an infinite set of fields depending on the spatial coordinate $x$ and the time parameters $t_{k}$. The infinite set of differential equations for the fields $U_{i}$ is introduced via the equations

$$
\frac{\partial L}{\partial t_{k}}=\left[L_{+}^{k}, L\right]
$$

where + denotes the purely differential part of the k-th power of the Lax operator. The quantities

$$
F_{k}=\frac{1}{k} \operatorname{Res} L^{k}
$$

are first integrals of motion for the flows (37). Here res denotes the integral Res $A=$ $\int d x a_{-1}$ for the generic pseudodifferential operator $A=\ldots+a_{-1} \partial^{-1}+\ldots$.

For the Nonlinear Schrödinger Equation we choose the scalar Lax pair [17] as

$$
L=\partial_{x}+J_{-} \partial^{-1} J_{+},
$$

and our equation could be written down as

$$
\dot{L}=\left[L_{+}^{2}, L\right] .
$$

For the extended $(\mathrm{N}=2)$ supersymmetric case we consider the super pseudodifferential Lax operator $[9,10]$ in the form $(36)$ where now

$$
U_{i}=b 1_{n}+f 1_{n} \mathcal{D}_{1}+f 2_{n} \mathcal{D}_{2}+b 2_{n} \mathcal{D}_{1} \mathcal{D}_{2},
$$

and the $b 1$ and $b 2$ are the superbosons while $f 1$ and $f 2$ are superfermions. In order to find the supersymmetric Lax pair which gives us the Superextension of the Nonlinear Schrödinger Equation we can postulate the most general form of this pair and try to fix it by computing the equality (40). We used the symbolic manipulation program REDUCE and found that the following Lax operator

$$
L=\partial_{x}+G \cdot \partial^{-1} \cdot \mathcal{D}_{1} \mathcal{D}_{2} F,
$$

gives us the equations (10-11).

This Lax operator could be reduced to the bosonic case or to the nonextended case. For the first case we obtained

$$
L=\left(\begin{array}{cc}
\partial_{x}+g \partial^{-1} f, & g \partial^{-1} j_{+} \\
-g \cdot \partial_{x} j_{+}+j_{-} \partial^{-1} f, & \partial_{x}+j_{-} \partial^{-1} j_{+}
\end{array}\right),
$$


which gives us the equations (12-15). For the second case we have

$$
L=\left(\begin{array}{cc}
\partial_{x}-\eta \partial^{-1} \zeta, & \eta \partial^{-1} j_{+} \\
-j_{-} \partial^{-1} \zeta, & \partial_{x}+j_{-} \partial^{-1} j_{+}
\end{array}\right),
$$

with the equations (24-27). First, let us present four nontrivial conservation laws for our model. In order to do it we modify the formula (38) for which

$$
A=\ldots+b 2_{-1} \mathcal{D}_{1} \mathcal{D}_{2} \partial^{-1}+\ldots
$$

and therefore $a_{-1}=b 2_{-1}$,

$$
\begin{aligned}
H_{1}= & \int d X G \cdot F, \\
H_{2}= & \int d X G_{x} F, \\
H_{3}= & \int d X\left\{G_{x x} F-\frac{1}{3} G^{3} F^{3}+G F\left(\mathcal{D}_{1} \mathcal{D}_{2}(G F)\right)\right\}, \\
H_{4}= & \int d X\left\{G_{x x x} F+\frac{1}{2} G^{2} F^{2}\left(G F_{x}-G_{x} F\right)+\right. \\
& \left.\frac{3}{2}\left(G_{x} F-G F_{x}\right)\left(\mathcal{D}_{1} \mathcal{D}_{2}(G \cdot F)\right)\right\},
\end{aligned}
$$

where now $d X=d x d \Theta_{2} d \Theta_{1}$ and our integral is understand as the Berezin integral.

Finally let us discuss the hamiltonian formulation of our supersymmetric extension. Our supersymmetric extension can be written down as the

$$
\frac{d}{d t}\left(\begin{array}{l}
F \\
G
\end{array}\right)=P_{1} \cdot \operatorname{grd} H_{3}
$$

where, interestingly the Poisson tensor has the same form as in (31). This formulation gives us the first hamiltonian structure. Unfortunately we could not find its second structure, what is probably connected with the nonexistence of the $\mathrm{N}=2$ supersymmetric $\operatorname{sl}(2)$ Kac-Moody algebra.

\section{References}

[1] C.S. Gardner, J.M. Green, M.D. Kruskal and R.M. Miura, Phys. Rev. Lett. 19 (1967) 1095.

[2] L.A. Dickey, Soliton Equation and Hamiltonian Systems, Adv. Serie Phys. vol. 12, World Scientific 1991.

[3] L.D. Faddeev and L.A. Takhtajan, "Hamiltonian Methods in the Theo Solitons" (Springer, Berlin,1987).

[4] M. Ablowitz and H. Segur, "Solitons and the Inverse Scattering Tr SIAM, Philadelphia 1981. 
[5] B.A. Kupershmidt, "Elements of Superintegrable Systems" Reidel, Dordrecht, 1987.

[6] M. Chaichian and P. Kulish, Phys. Lett. B 183 (1987) 169.

[7] P.P. Kulish, Lett. Math. Phys. 10 (1985) 87-93.

[8] M. Chaichian and J. Lukierski, Phys. Lett. B 212 (1989) 461.

[9] C.A. Laberge and P. Mathieu, Phys. lett. B 215 (1988) 718.

[10] W. Oevel and Z. Popowicz, Comm. Math. Phys. 139, 441 (1991

[11] Z. Popowicz, Phys. Lett. A 174 (1993) 411.

[12] E. Ivanow and S. Krivonos, Phys. Lett. B 291 (1992) 63.

[13] Z. Popowicz, Phys. Lett. B 319 (1993) 478.

[14] G.H.M. Roelofs and P.H.M. Kersten, J. Math. Phys. 33 (1992) 2185.

[15] R.A. Chowdhury and M. Naskar, J. Math. Phys. 28 (1987) 1809.

[16] J.C. Brunelli and A. Das, "Test of Integrability of the Supersymm Nonlinear Schrödinger Equation" Preprint UR-1344, hep-th 9403019.

[17] F. Toppan, "N=1, 2 Super-NLS Hierarchies as Super-KP Coset Reduct Preprint Enslapp -l-467/94, hep-th 9405095.

[18] V.E. Zakharov and A.B. Shabat, Sov. Phys. JETP 34 (1972) 62.

[19] O. Ragnisco and W. Oevel, Physica A 161 (1989) 181. 